\title{
ON THE DIFFRACTION OF LIGHT BY AN OBLIQUELY HELD
} CYLINDER.

By $\mathrm{T}$. $\mathrm{K}$. Chinmayanandam.

\section{INTRODUCTION.}

THE problem of the diffraction of light by a transparent cylinder, on which plane waves are normally incident, has been treated by many writers, ${ }^{1}$ both with respect to the classical wave theory, and from the fundamental electro-magnetic equations, the problem having special application to the theory of the rainbow. The writer has observed some very interesting phenomena, which are exhibited when the light falls obliquely on the cylinder, and which do not seem to have been previously described. It is proposed in this paper to give an account of the phenomena, and to attempt a theoretical explanation.

If light falls on a transparent cylinder normally, the rays that emerge after refraction and one internal reflection form, as is well known, two parallel caustics whose directions make a definite angle, which, for glass, is about $45^{\circ}$. If the incidence of light on the cylinder is made oblique, the two caustics are found to approach each other more and more closely. At a certain angle of incidence (about $50^{\circ}$, for glass), the two caustics of the emergent rays coalesce, and absolutely cease to exist for greater angles of incidence. At and near the stage where the caustics and the supernumerary fringes, which accompany them, coalesce, the phenomena presented are of particular interest, and may be studied by mounting the cylinder horizontally on the table of a spectrometer. The collimator slit should also be made horizontal, and may be illuminated by the electric arc or by a Cooper-Hewitt lamp. With this arrangement, we can observe visually or photograph the phenomena corresponding to varying angles of incidence simultaneously. Fig. I reproduces a photograph of the effects thus observed in monochromatic light. If part of the length of the horizontal slit of the collimator be covered up, so as to leave only a short

${ }^{1}$ For detailed references to the earlier work by Airy, Mascart, Pernter, Miller, Pulfrich, and others, see a paper by Aichi and Tanakdate on the "Theory of the Rain-bow, Due to a Circular Source of Light," Journ. of the Coll. of Sc., Imp. Univ. of Tokyo, Vol. XXI., Art. 3 (I906). Among recent references, may be mentioned Debye, Phys. Zeitschr., 9, Nov., I908, also Sc. abstracts, I909, p. 88, and Mobius "On the Theory of the Rain-bow," Ann. der Phy., 33 (I9I0), and 40 (I9I3). See also Potzger on "Diffraction in the Ultra-microscope," Ann. der Phys., 30 (1909). 
opening, the phenomena corresponding to any particular angle of incidence are observed in the field. In the photograph shown, they would correspond to a narrow strip perpendicular to the bisector of the angle between the two bright caustic lines.

The interesting feature of the diffraction pattern, which distinguishes it from the fringes in the rainbow, is that the two systems of caustic fringes, which are far apart in the case of normal incidence, overlap and interfere with each other when the incidence is oblique, giving rise to a beautiful structure in the fringe-system, especially near its termination, where the caustics meet and vanish. As the angular separation of the caustics increases, the overlapping of the fringes becomes less and less perceptible in white light, but a few fine interference fringes, which form practically a separate system, may be seen midway between the caustics; with the spectrometer, these are visible even at an incidence nearly normal to the cylinder, if its diameter is about $0.5 \mathrm{~mm}$. or less. When the diameter of the cylinder is of the order of o.or $\mathrm{mm}$., these fringes become broad and bright. In this case, the caustics and their supernumeraries are practically achromatic, but the system of interference fringes seen midway between the caustics is strongly colored, except, of course, the central fringe which is white. Figs. $2(a, b$, and $c)$ represent the phenomena observed with a glass fiber of that diameter, and correspond respectively to three different angles of incidence.

\section{Geometrical Theory.}

Let the axis of the cylinder be the $z$-axis, the incident rays being parallel to the $x z$-plane. The direction cosines of the incident ray may be taken to be $(l, o, n)$, and the point of incidence of any ray on the cylinder may be represented by the cylindrical coördinates $(a \cos \varphi$, $a \sin \varphi, z), a$ being the radius of the cylinder. Also let the direction cosines of the refracted ray, the internally reflected ray, and the emergent ray be respectively $\left(l^{\prime}, m^{\prime}, n^{\prime}\right),\left(l^{\prime \prime}, m^{\prime \prime}, n^{\prime \prime}\right)$, and $(\lambda, \mu, \nu)$. Then we shall have, for the first refracted ray, from the fundamental laws of refraction,

and

$$
\left|\begin{array}{ccc}
l, & o, & n \\
\cos \varphi, & \sin \varphi, & o \\
l^{\prime}, & m^{\prime}, & n^{\prime}
\end{array}\right|=0 .
$$

nd

$$
\mathbf{I}-l^{2} \cos ^{2} \varphi=\mu^{2}\left\{\mathbf{I}-\left(l^{\prime} \cos \varphi+m^{\prime} \sin \varphi\right)^{2}\right\} .
$$

and we shall have two similar pairs of equations for the other two rays. Solving equations (I) and (2), we get 
where

$$
\left.\begin{array}{rl}
l^{\prime} & =-k \cos \varphi+\frac{l}{\mu} \sin ^{2} \varphi \\
m^{\prime} & =-k \sin \varphi-\frac{l}{\mu} \sin \varphi \cos \varphi \\
n^{\prime} & =\frac{n}{\mu}
\end{array}\right\}
$$

$$
k^{2}=\mathrm{I}-\frac{\mathrm{I}}{\mu^{2}}\left(\mathrm{I}-l^{2} \cos ^{2} \varphi\right)
$$

If this refracted ray meets the surface of the cylinder at a point $\varphi^{\prime}$, we have

$$
\frac{\cos \varphi^{\prime}-\cos \varphi}{l^{\prime}}=\frac{\sin \varphi^{\prime}-\sin \varphi}{m^{\prime}}
$$

from which, using equations $(A)$, we can deduce the relation

$$
\tan \frac{\varphi-\varphi^{\prime}}{2}=\frac{k \mu}{l \sin \varphi}
$$

Similarly, if the point of emergence of the ray from the surface of the cylinder has the coördinate $\varphi^{\prime \prime}$, it can be shown that

and also that

$$
\tan \frac{\varphi^{\prime}-\varphi^{\prime \prime}}{2}=\frac{k \mu}{l \sin \varphi}=\tan \frac{\varphi-\varphi^{\prime}}{2}
$$

and

$$
\left.\begin{array}{l}
l^{\prime \prime}=-k \cos \varphi^{\prime}+\frac{l}{\mu} \sin \varphi \sin \varphi^{\prime} \\
m^{\prime \prime}=-k \sin \varphi^{\prime}-\frac{l}{\mu} \sin \varphi \cos \varphi^{\prime} \\
n^{\prime \prime}=\frac{n}{\mu}
\end{array}\right\}
$$

$$
\left.\begin{array}{l}
\lambda=l \cos \left(\varphi+\varphi^{\prime \prime}\right)=l \cos 2 \varphi^{\prime} \\
\mu=l \sin \left(\varphi+\varphi^{\prime \prime}\right)=l \sin 2 \varphi^{\prime} \\
\nu=n
\end{array}\right\} .
$$

Equations $(C)$ determine the direction of the emergent rays. If the radius of the cylinder is very small, we can regard, as an approximation, that the rays all start from the origin $o$ itself. Then the emergent rays will lie on the cone

$$
x^{2}+y^{2}=\frac{l^{2}}{n^{2}} z^{2}
$$

They would be parallel when 
Physical Review, Vol. XII., Second Series. October, I9I8.
Plate I.

To face page 316

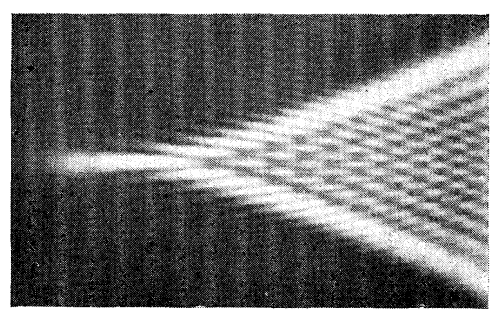

Fig. 1.

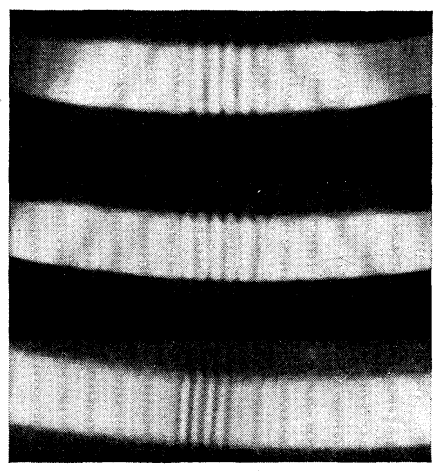

Fig. 2.

T. K. CHINMAYANANDAM. 


$$
\frac{d \lambda}{d \varphi}=0, \quad \frac{d \mu}{d \varphi}=0,
$$

which reduce to the condition that

From (4), we have,

$$
\frac{d \varphi^{\prime}}{d \varphi}=0
$$

$$
\begin{aligned}
& \cos \left(\varphi-\varphi^{\prime}\right)=\frac{l^{2} \sin ^{2} \varphi-\mu^{2} k^{2}}{\mu^{2}-\mathbf{I}+l^{2}}, \\
& \sin \left(\varphi-\varphi^{\prime}\right)=\frac{4 l^{2} \sin \varphi \cos \varphi}{\mu^{2}-\mathrm{I}+l^{2}}, \quad \text { since } \frac{d \varphi^{\prime}}{d \varphi}=0 \\
& =\frac{2 \mu k l \sin \varphi}{\mu^{2}-\mathbf{I}+l^{2}} \\
& \text { by (4). }
\end{aligned}
$$

Hence

$$
\cos \varphi=\frac{\mu k}{2 l}
$$

or by (3),

$$
\cos ^{2} \varphi=\frac{\mu^{2}-\mathrm{I}}{3 l^{2}} .
$$

If the incidence is normal, $l^{2}=\mathrm{I}$ and equation (6) reduces to

$$
\cos ^{2} \varphi=\frac{\mu^{2}-\mathrm{I}}{3},
$$

which is the condition for crowding of the emergent rays from a rain-drop. It is also seen from (6) that $\varphi$ will be imaginary, if $l^{2}<\left(\mu^{2}-\mathrm{I}\right) / 3$, which happens for glass if the angle of incidence is greater than about $50^{\circ}$. The caustics, therefore, cease to exist at very oblique incidence.

The actual direction of the emergent rays, where they are parallel, $i . e .$, the direction of the cuastics may now be determined.

$$
\begin{aligned}
\cos 2 \varphi^{\prime} & =\cos \left\{2\left(\varphi-\varphi^{\prime}\right)-2 \varphi\right\} \\
& =\frac{\mathrm{I}}{\left(\mu^{2}-\mathrm{I}+l^{2}\right)^{2}}\left\{\mathrm{I} 2 l^{2} s+3 s^{2}-l^{4}+\frac{2 s^{3}}{l^{2}}\right\}
\end{aligned}
$$

from equations (4) and (6), $s$ being written for $\left(\mu^{2}-1\right) / 3$. The directions of the two caustics are thus given by

$$
\left.\begin{array}{rl}
\lambda & =l \cos 2 \varphi^{\prime} \\
\mu & = \pm l \sin 2 \varphi^{\prime} \\
\nu & =n
\end{array}\right\},
$$

where $\varphi^{\prime}$ is determined by (8). The angle between these two directions is given by 


$$
\cos \epsilon=\mathrm{I}-2 l^{2} \sin ^{2} 2 \varphi^{\prime} .
$$

The results of a few measurements of the angular separation of the caustics, at varying angles of incidence, are given below, with the corresponding values calculated from theory. The experimental arrangement used was simply to have the cylinder mounted on the table of a spectrometer, making the collimator slit short and narrow. The electric arc was used with a green ray filter for illuminating it. The angle of incidence was measured, as usual, by setting the cross-wire of the telescope on the reflected image of the slit, and then taking the direct reading. The angle between the caustics was determined by reading off the linear separation in $\mathrm{cm}$. of the caustics, on a screen held at a distance of one meter from the cylinder, and dividing it by roo. The refractive index of the cylinder was measured by dipping it in a rectangular glass cell, illuminated by green light and containing Thoulet's solution. ${ }^{1}$ The liquid, whose refractive index could be continuously varied by mixing water, was diluted until the shadow of the cylinder became practically invisible. The chief difficulty in the experiment was the selection of the cylinder itself. Most of the large number of glass threads that were examined gave somewhat irregular effects, a slight rotation of the cylinder about its own axis changing the angle between the emergent caustics very much. The irregularities are apparently due to the cylinder being not quite circular, i. e., to slight ellipticity in its cross-section. Considering the fact that even a slight ellipticity in the cross-section of the cylinder affects the results appreciably, the agreement between the observed and calculated values is good. As was anticipated from theory from equation (7), the caustics cease to exist after a critical angle of incidence, which is about $50^{\circ}$ here.

TABLE I.

\begin{tabular}{c|c|c}
\hline \multirow{2}{*}{ Angle of Incidence. } & \multicolumn{2}{|c}{ Angular Separation of the Caustics. } \\
\cline { 2 - 3 } & Observed. & Calculated. \\
\hline $32^{\circ} 15^{\prime}$ & $17^{\circ} 12^{\prime}$ & $17^{\circ} 48^{\prime}$ \\
$35^{\circ} 15^{\prime}$ & $13^{\circ} 42^{\prime}$ & $13^{\circ} 47^{\prime}$ \\
$38^{\circ} 15^{\prime}$ & $10^{\circ} 30^{\prime}$ & $10^{\circ} 0^{\prime}$ \\
$41^{\circ} 15^{\prime}$ & $6^{\circ} 54^{\prime}$ & $7^{\circ} 18^{\prime}$ \\
$44^{\circ} 15^{\prime}$ & $3^{\circ} 42^{\prime}$ & $3^{\circ} 12^{\prime}$ \\
$47^{\circ} 15^{\prime}$ & $1^{\circ} 12^{\prime}$ & $1^{\circ} 50^{\prime}$ \\
$49^{\circ} 39^{\prime}$ & $0^{\circ}$ & $0^{\circ}$ \\
$52^{\circ}$ & & \\
\hline
\end{tabular}

$(\mu=1.51)$.

${ }^{1}$ See R. W. Cheshire, Phil. Mag., Oct., I916. 


\section{Form of the Emergent Wave-surface.}

Passing on now to consider the phenomena in the light of the wave theory, we have first to determine the form of the wave on emergence from the cylinder. Figs. 3 and 4 represent the projection of the wave

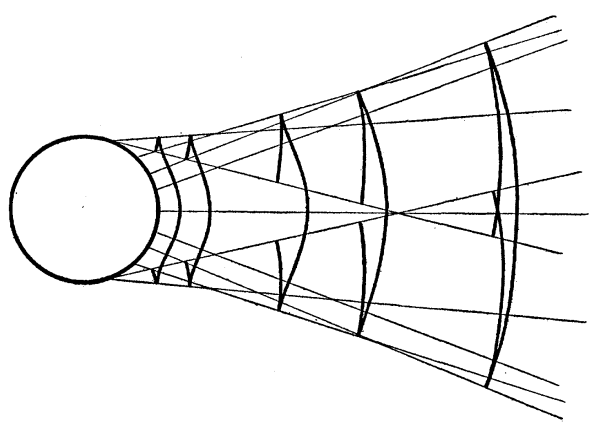

Fig. 3.

front on the plane passing through the two caustics, Fig. 3 illustrating the case of normal incidence, and Fig. 4, the case when the incidence is about $50^{\circ}$, the angle between the caustics being then almost zero; they have been drawn by plotting out a number of emergent rays with data calculated from the formula in section 2 above, and drawing curves normal to them. It will be seen that the section of the emergent wave has two points of inflection on either side of the central line, and at a

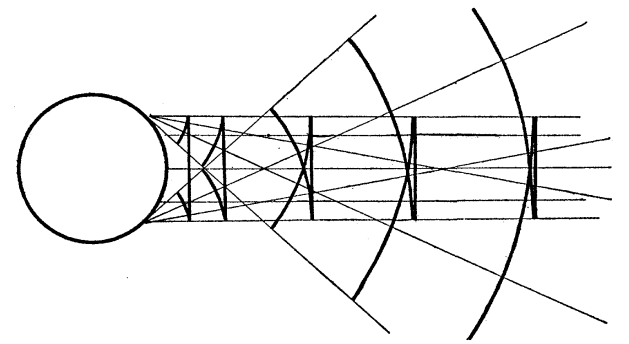

Fig. 4.

great distance from the cylinder, the disturbance at any point may be regarded as due to the interference of three sets of wave trains, to which the emergent cusped wave gives rise. In a direction close inside either of the caustics, the effect of one of the wave-trains is negligible in comparison with that of the other two, and the problem of determining the illumination in any direction is then the same as in the case of the rainbow, which has been worked out by Airy, Mascart, and others. But the 
approximation suggested is not valid in directions nearly midway between the caustics, even when the latter are widely separated, and is totally inapplicable when the caustics make only a small angle with each other. This is evident on an inspection of Fig. I. At a sufficient distance from the "tail" of the pattern, the caustics are widely separated and the fringe system is much like the supernumerary fringes in the rainbow, except near the central line where, as already explained, some interference fringes are observed. But near the "tail," a peculiar criss-cross structure is observed in the fringe-system.

\section{Calculation of the Intensity of Illumination.}

It follows from equation 5 in section 2 above that the emergent wave may be regarded approximately as spreading on the surface of a cone, whose axis coincides with that of the cylinder. But if the angular separation of the caustics is small, we can make a further approximation and regard the wave as spreading entirely on planes parallel to that passing through the two caustics. The section of the wave-front by that plane may, in effect, be considered to be a symmetrical curve of the form $O D O^{\prime}$ shown in Fig. 5, with two inflection points $O, O^{\prime}$ on either side, the normals at those two points indicating the direction of the emergent rays where they are parallel. In any direction inclined at a small angle $\theta$ to one of the normals, it is apparent from the figure that there are three rays originating at three different points $a, b, c$ on the emergent wave. We can consider the effect at any point in the direction $\theta$ as due to the interference of these three rays emerging from the wavefront in that direction. We can, for simplicity, regard the amplitude of disturbance to be the same at all points on the emerging wave surface itself. Then the amplitudes of the three rays which proceed in the direction $\theta$, will, at a great distance from the cylinder, be proportional to the square roots of the radii of curvature of the wave $O D O^{\prime}$ (Fig. 5) at the points $a, b, c$ from which they respectively originate. We have next to calculate the path difference of each pair of these rays, and then the illumination at a point can be easily calculated.

A simple method of analytically representing the curve $O D O^{\prime}$ suggests itself. The curve may be regarded as two curves $O D, D O^{\prime}$ fitted up to form one continuous curve, the equation to the former being

$$
y_{1}=A x_{1}{ }^{3}
$$

with respect to $O$, the inflection point, as origin, and the inflectional tangent $O X_{1}$, and normal $O Y_{1}$ as axes of reference, and the equation to the latter being

$$
Y_{2}=A x_{2}{ }^{3}
$$


with respect to $O^{\prime}$ as origin, and the inflectional tangent and normal at that point as axes of reference. The curves are considered as extending to infinity on the positive side of $x$, but are obviously limited on the negative side by the central line $C C^{\prime}$.

Consider first the rays that start out from the points $a$ and $b$. The amplitudes of the disturbances due to them are by symmetry equal, and will be proportional to $a_{1}$ where

$$
a_{1}^{2}=\mathrm{I} / \frac{d^{2} y}{d x^{2}}=\mathrm{I} / 6 A x= \pm \mathrm{I} / 6 A\left(\frac{\theta}{3 A}\right)^{1 / 2}
$$

Taking into account the gain in phase of $\pi / 2$ (indicated by the negative sign) of one of the rays, which passes through a focus, we find that the amplitude of either ray is

$$
a_{1}=(\mathrm{I} 2 A \theta)^{-1 / 4} \text {. }
$$

The phase difference between the two rays can be shown to be equal to

$$
\frac{2 \pi}{\lambda}\left[\frac{4}{3} \theta\left(\frac{\theta}{3 A}\right)^{1 / 2}\right]-\frac{\pi}{2} .
$$

The amplitude of the resultant of the two will be

$$
a_{2}=2(\mathrm{I} 2 A \theta)^{-1 / 4} \cos \left\{\frac{\pi}{\lambda} \cdot \frac{4}{3} \theta\left(\frac{\theta}{3 A}\right)^{1 / 2}-\frac{\pi}{4}\right\}
$$

and its phase will be $[-(\pi / 4)]$ with respect to the ray from the center $O$ in the same direction. We have now to determine the amplitude $a_{3}$ of the ray from the point $c$, and its phase $\delta$ with respect to the resultant of the first two rays. If the angle between $O y_{1}$ and $O^{\prime} y_{2}$ be denoted by $2 \alpha$,

$$
a_{3}=\{\mathrm{I} 2 A(2 \alpha-\theta)\}^{-1 / 4} \text {. }
$$

The perpendicular from $O$ on the tangent to the curve $D O^{\prime}$ at the point $c$ can be shown to be

$$
\begin{array}{r}
\frac{\mathrm{I}}{\left\{\mathrm{I}+(2 \alpha-\theta)^{2}\right\}^{1 / 2}}\left[2(2 \alpha-\theta)\left(\frac{\alpha}{3 A}\right)^{1 / 2}-\alpha\left(\frac{\alpha}{3 A}\right)^{1 / 2}+\frac{2}{3}(2 \alpha-\theta)\left(\frac{2 \alpha-\theta}{3 A}\right)^{1 / 2}\right] \\
=\left(\frac{\alpha}{3 A}\right)^{1 / 2}(3 \alpha-2 \theta)+\frac{2}{3}(2 \alpha-\theta)\left(\frac{2 \alpha-\theta}{3 A}\right)^{1 / 2} \text { (approx.). }
\end{array}
$$

Hence, allowing for the gain in phase of $\pi / 2$ of the third ray in passing through a focus, we get

$$
\delta=\frac{2 \pi}{\lambda}\left[\left(\frac{\alpha}{3 A}\right)^{1 / 2}(3 \alpha-2 \theta)+\frac{2}{3}(2 \alpha-\theta)\left(\frac{2 \alpha-\theta}{3 A}\right)^{1 / 2}\right]-\frac{\pi}{4} .
$$

The illumination in the direction $\theta$ is then given by

$$
I=a_{2}^{2}+a_{3}^{2}+2 a_{2} a_{3} \cos \delta
$$

$a_{2}, a_{3}$ and $\delta$ being determined by equations (II), (I2) and (I3). 
Illumination Outside the Caustics.-It is seen from (I I) that $a_{2}$ becomes infinite when $\theta=0$, and also that the above theory is inapplicable when $\theta$ becomes negative. For, outside the caustics, it is apparent that there is only one ray which emerges in any given direction. But, since the emergent cone of light is abruptly terminated in the direction $\theta=0$, we must also take into account a diffraction effect, similar to that within the geometrical shadow of a straight edge. According to Sommerfeld's well-known investigation, ${ }^{1}$ this effect can be represented by that due to a single radiating source placed at the edge, $i$. e., at $O$ (Fig. 5) in our

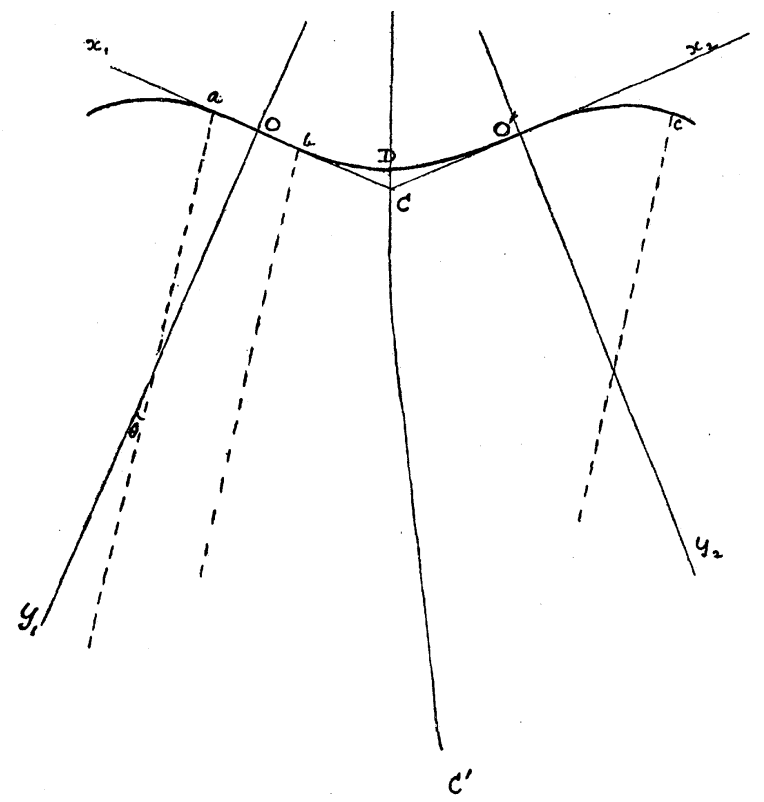

Fig. 5.

problem. Since the whole effect is small, we can consider approximately that the diffracted ray is of the same amplitude as the ray which emerges in the same direction from the other half of the wave-front. The illumination curves, outside the caustics, have been actually drawn on this assumption, the curves being rounded off at the point corresponding to $\theta=0$.

The value of the constant $A$ can be calculated approximately by equating the length $O D$ (Fig. 5) to $\left(a \sin \varphi^{\prime \prime}\right)$, where $\varphi^{\prime \prime}$ corresponds to the point of emergence of the caustic from the surface of the cylinder. Thus we shall have

${ }^{1}$ Sommerfeld, On the Math. Theory of Diffraction, Math. Annalen, Vol. XLVII., p. 3I7 (I895). 


$$
\left(\frac{\alpha}{3 A}\right)^{1 / 2}=a \sin \varphi^{\prime \prime} \text { (approx.). }
$$

The intensity of illumination has been calculated on the above theory for different values of $\theta$ and the illumination curves, for the cases when the angle between the caustics $(2 \alpha$, approximately) is (I) $\mathrm{o}^{\circ} 56^{\prime}$, (2) $\mathrm{I}^{\circ} 52^{\prime}$, are shown in Fig. 6. ${ }^{1}$ A comparison of the curves with the photograph (Fig. I) shows that the theory is capable of explaining the facts very closely. To make the comparison quantitative, a particular section, perpendicular to the bisector of the angle between the caustic lines, was chosen so that the general form of the illumination curve was practically the same as that obtained by calculation,
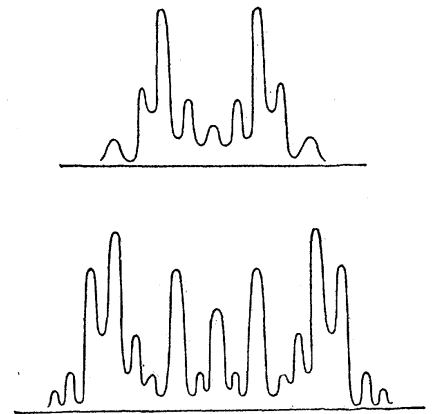

Fig. 6. and then the positions of the successive maxima with respect to the central line $c c^{\prime}$ (Fig. 5) were measured. The calculated and observed values are tabulated below for comparison, for the two cases for which values have been calculated.

TABLE II.

\begin{tabular}{|c|c|c|c|c|c|c|}
\hline \multirow{3}{*}{$\begin{array}{c}\text { Serial No. } \\
\text { of Max. } \\
\text { (Reck- } \\
\text { oned from } \\
\text { the Cen- } \\
\text { tral Direc- } \\
\text { tion). }\end{array}$} & \multicolumn{3}{|c|}{ Separation of Caustics $0^{\circ} 5^{6^{\prime}}$} & \multicolumn{3}{|c|}{$x^{\circ} 52^{\prime}$} \\
\hline & \multirow{2}{*}{$\begin{array}{l}\text { Calc. Inten- } \\
\text { sity. }\end{array}$} & \multicolumn{2}{|c|}{ Position of Max. } & \multirow{2}{*}{$\begin{array}{l}\text { Calc. Intensity } \\
\text { of Max. }\end{array}$} & \multicolumn{2}{|c|}{ Position of Max. } \\
\hline & & Obsd. & Calc. & & Obsd. & Calc. \\
\hline 1 & 1.6 & $0^{\circ} 10^{\prime}$ & $0^{\circ} 11^{\prime}$ & 0.5 & $0^{\circ} \quad 8^{\prime}$ & $0^{\circ} \quad 8^{\prime}$ \\
\hline 2 & 4.0 & $0^{\circ} 21^{\prime}$ & $0^{\circ} 21^{\prime}$ & 1.7 & $0^{\circ} 17^{\prime}$ & $0^{\circ} 18^{\prime}$ \\
\hline 3 & 2.0 & $0^{\circ} 31^{\prime}$ & $0^{\circ} 30^{\prime}$ & 0.4 & $0^{\circ} 26^{\prime}$ & $0^{\circ} 29^{\prime}$ \\
\hline 4 & 0.7 & $0^{\circ} 41^{\prime}$ & $0^{\circ} 41^{\prime}$ & 0.9 & $0^{\circ} 33^{\prime}$ & $0^{\circ} 36^{\prime}$ \\
\hline 5 & & & & 2.2 & $0^{\circ} 43^{\prime}$ & $0^{\circ} 43^{\prime}$ \\
\hline 6 & & & & 1.7 & $0^{\circ} 53^{\prime}$ & $0^{\circ} 54^{\prime}$ \\
\hline 7 & & & & 0.5 & $1^{\circ} 4^{\prime}$ & $1^{\circ} 3^{\prime}$ \\
\hline 8 & & & & 0.3 & $1^{\circ} 13^{\prime}$ & $1^{\circ} 10^{\prime}$ \\
\hline
\end{tabular}

The agreement between the observed and calculated values is fairly close. It is also noteworthy that the maxima and minima seem to be ranged at roughly equal intervals.

Some explanation may now be given of the peculiarity of the phenomena, described in section ( $\mathrm{r}$ ), observed when the diameter of the

1 The abscissæ are on the same scale in the two diagrams. 
cylinder is very small. In a direction midway between the caustics, it may be noted that the rays emerging from points $a$ and $c$ (Fig. 5) have nearly equal paths, while the central ray from $b$ differs very much in path from either of the other two. Hence we may regard the phenomena in white light as due simply to the superposition of a practically uniform illumination (due to the ray from $b$ ) on a fringe system due to interference of light from the two point sources $a$ and $c$. It would be seen from Fig. 2 that the central fringes are very distinct and bright, and this would indicate that the effect due to the ray from $b$ is much smaller than that due to those from $a$ and $c$. This seems to be what actually happens, for, as a reference to Figs. 3 and 4 will show, there is in fact a greater concentration of the rays near the points $a$ and $c$ than at the center of the wave front. The achromatism of the caustics and their supernumeraries ${ }^{1}$ is due to the fact that the effect of dispersion in the glass is compensated by that of diffraction of the emergent wave.

The work was carried out in the laboratory of the Indian Association for the Cultivation of Science. The writer wishes to acknowledge the interest taken in this investigation by Prof. C. V. Raman.

${ }^{1}$ See Mascart, Ann. de Chim. et de Phys. (6), 26 (I892). 


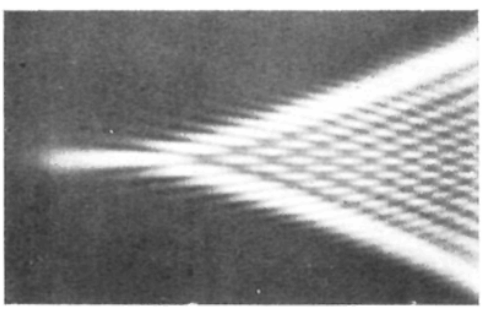

Fig. 1.

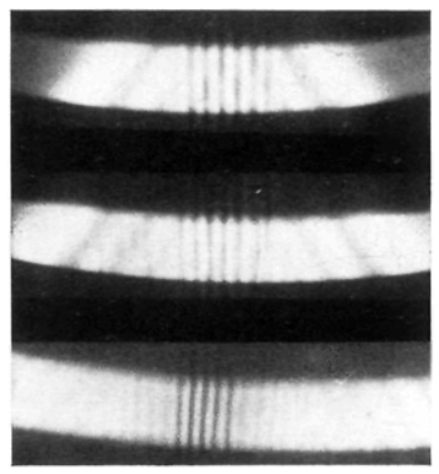

Fig. 2. 